\title{
FILTERING COHOMOLOGY AND LIFTING VECTOR BUNDLES
}

BY

\author{
E. GRAHAM EVANS AND PHILLIP GRIFFITH ${ }^{1}$
}

\begin{abstract}
For a module $M$ over a local Cohen-Macaulay ring $R$ we develop a (finite) sequence of presentations of $M$ which facilitates the study of invariants arising from the cohomology modules of $M$. As an application we use this data, in case $R$ is regular and $M$ represents a vector bundle on the punctured spectrum of $R$ with a vanishing cohomology module, to obtain bounds on how far $M$ can be lifted as a vector bundle.
\end{abstract}

In an earlier article [5] we used the dual of a filtration of Auslander and Bridger [1] to obtain some results for nontrivial $k$ th syzygies of rank $k$. In this article we establish a different method for filtering a module over a local Cohen-Macaulay ring, or more precisely a method for filtering a free presentation of a module (see discussion following Theorem 1.8). This filtration is accomplished through a finite sequence of presentations.

To be specific, let $R$ be a local Cohen-Macaulay ring and let $M$ be a finitely generated $R$-module such that $\operatorname{depth}\left(\operatorname{Ext}^{i}(M, R)\right) \geqslant i$ for all $i$ less than or equal to the dimension of $R$. In Theorem 1.2 we establish an exact sequence, which we term a $q$ th presentation of $M$,

$$
0 \rightarrow \mathscr{K}_{q}(M) \rightarrow \mathscr{E}_{q}(M) \rightarrow M \rightarrow 0
$$

such that the projective dimension of $\mathscr{K}_{q}(M)$ is less than $q$ and such that $\operatorname{Ext}^{i}\left(\mathscr{E}_{q}(M), R\right)$ is zero for $i$ from one through $q$. In addition we establish uniqueness results (see Corollary 1.6 and Theorem 1.7) in the sense of stable isomorphism. As a consequence we recapture a variation of our main result in [6] without the assumptions on rank or the existence of maximal Cohen-Macaulay modules. We also show, if $R$ is Gorenstein, that the intermediate cohomology of a module is always carried by a module of finite projective dimension (see Corollary 1.9).

In $\$ 2$ we turn our attention to the lifting of vector bundles on the punctured spectra of regular local rings and achieve a substantial improvement over our results in [5]. In particular, if $\rho_{i}(M)$ denotes the rank of the $i$ th syzygy of $\operatorname{Ext}^{i}(M, R)$ for $i \geqslant 0$, we show that a nontrivial vector bundle $M$ with $\operatorname{Ext}^{q}(M, R)$ zero for some $q$ in the range $1 \leqslant q \leqslant \operatorname{dim} R-2$, cannot be lifted more than $\sum_{i=0}^{q-1} \rho_{i}(M)$ times. For a complete discussion of the projective case the reader should consult Barth and Van de Ven [2], Sato [12] and Tyurin [14]. For additional information on this problem

Received by the editors June 20, 1984.

1980 Mathematics Subject Classification. Primary 13C05, 13D03, 14 F03.

${ }^{1}$ Both authors were partially supported by the National Science Foundation during the preparation of this article. 
one should consult Hartshorne [7], our papers [4, 5] and the fundamental paper [9] by Horrocks.

1. The filtration. Serre in [13] and later Murthy in [11] use the following construction. Let $M$ be a finitely generated $R$-module of projective dimension one. Then there is an exact sequence

$$
0 \rightarrow R^{n} \rightarrow E \rightarrow M \rightarrow 0
$$

in which the map $\operatorname{Hom}\left(R^{n}, R\right) \rightarrow \operatorname{Ext}^{1}(M, R)$ is surjective. One concludes that $\operatorname{Ext}^{1}(E, R)$ is zero and hence, since the projective dimension of $E$ is at most one, that $E$ is projective. If one drops the requirement that $M$ has projective dimension one, then one can nevertheless construct such a sequence where $\operatorname{Ext}^{1}(E, R)$ is zero. This is the case $q=1$ of our construction. We utilized this construction implicitly (for $q=1$ ) in our article with Bruns [3, Lemma 2.1]. We present the full details here in order to give the flavor of our subsequent construction.

LEMMA 1.1. Let $R$ be a local ring and let $M$ be a finitely generated $R$-module with $\operatorname{Ext}^{1}(M, R)$ generated by $n$ elements. Then there is an exact sequence

$$
0 \rightarrow \mathscr{K}_{1}(M) \rightarrow \mathscr{E}_{1}(M) \rightarrow M \rightarrow 0
$$

such that $\mathscr{K}_{1}(M)$ is isomorphic to $R^{n}$ and such that $\operatorname{Ext}^{1}\left(\mathscr{E}_{1}(M), R\right)$ is zero.

Proof. If $n=0$ we take $\mathscr{E}_{1}(M)=M$ and $\mathscr{K}_{1}(M)=0$. If $n>0$ let $\xi_{1}, \ldots, \xi_{n}$ be a generating set for $\operatorname{Ext}^{1}(M, R)$ in which the class of $\xi_{i}$ is represented by the extension $0 \rightarrow R e_{i} \rightarrow N_{i} \rightarrow M \rightarrow 0$. We form the direct sum of these extensions and the pullback diagram

$$
\begin{array}{ccccccccc}
0 & \rightarrow & \oplus R e_{i} & \rightarrow & N & \rightarrow & M & \rightarrow & 0 \\
& \| & & \downarrow & & \downarrow \Delta & & \\
0 & \rightarrow & \oplus R e_{i} & \rightarrow & \oplus N_{i} & \rightarrow & M^{n} & \rightarrow & 0
\end{array}
$$

where $\Delta$ is the diagonal map. We note that the pushout of the top row along the $i$ th projection gives back $\xi_{i}$. Hence, the natural map $\operatorname{Hom}\left(\oplus \operatorname{Re}_{i}, R\right) \rightarrow \operatorname{Ext}^{1}(M, R)$ is surjective and therefore $\operatorname{Ext}^{1}(N, R)$ is zero. Thus with $\mathscr{E}_{1}(M)=N$ and $\mathscr{K}_{1}(M)=$ $\oplus R e_{i}$ we have the desired sequence.

THEOREM 1.2. Let $R$ be a Cohen-Macaulay local ring and let $M$ be a finitely generated $R$-module. Assume that the grade of $\operatorname{Ext}^{i}(M, R)$ is at least $i$ for each $i$ up to the dimension of $R$. Then for each $q$ between 1 and the dimension of $R$ there is a short exact sequence

$$
0 \rightarrow \mathscr{K}_{q}(M) \rightarrow \mathscr{E}_{q}(M) \rightarrow M \rightarrow 0
$$

such that the projective dimension of $\mathscr{K}_{q}(M)$ is less than $q$ and $\operatorname{Ext}^{i}\left(\mathscr{E}_{q}(M), R\right)$ is zero for $1 \leqslant i \leqslant q$.

Proof. We proceed by induction on $q$. The case $q=1$ is equivalent to Lemma 1.1. Let

$$
0 \rightarrow K \rightarrow F_{q-2} \rightarrow \cdots \rightarrow F_{1} \rightarrow F_{0} \rightarrow \mathscr{E}_{q-1}(M) \rightarrow 0
$$


be an exact sequence with the $F_{i}$ free $R$-modules and with $\mathscr{E}_{q-1}(M)$ already constructed with the desired properties. Then $\operatorname{Ext}^{q}(M, R)$ is isomorphic to $\operatorname{Ext}^{q}\left(\mathscr{E}_{q-1}(M), R\right)$. By a dimension shift we see that $\operatorname{Ext}^{q}(M, R)$ is isomorphic to $\operatorname{Ext}^{1}(K, R)$. We apply the case $q=1$ to $K$ and obtain a diagram

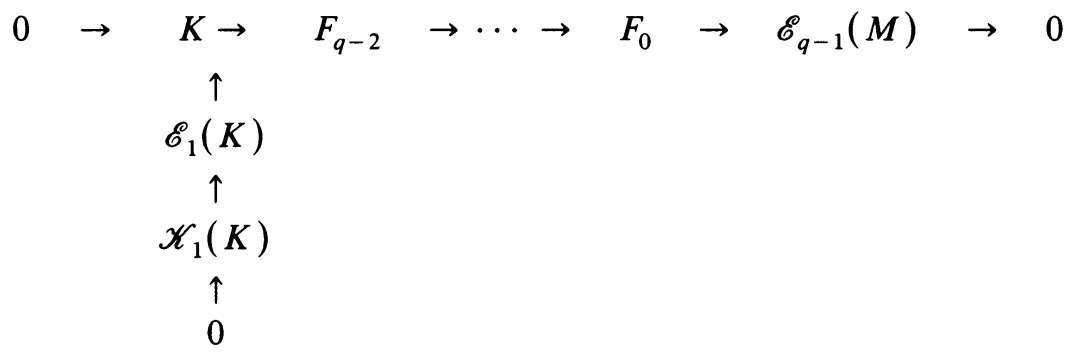

where $\mathscr{K}_{1}(K)$ is $R^{n}$, the integer $n$ being the number of generators of $\operatorname{Ext}^{1}(K, R)$. Dualizing with respect to $R$ and using that $\operatorname{Ext}^{i}\left(\mathscr{E}_{q-1}(M), R\right)=0$ for $1 \leqslant i \leqslant q-1$, we obtain the diagram

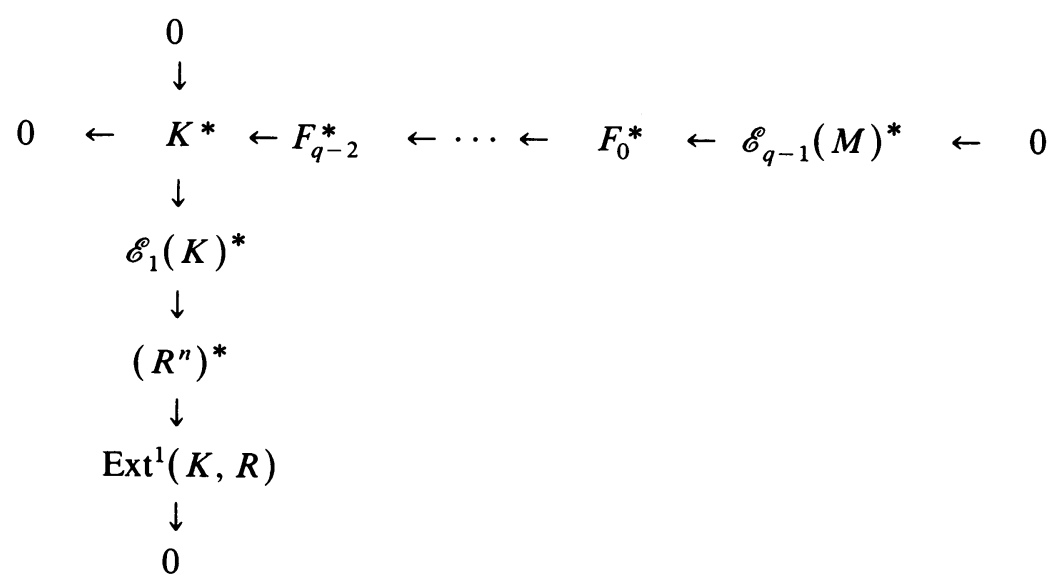

with exact row and column. Let $V$ be the image of $\mathscr{E}_{1}(K)^{*}$ in $\left(R^{n}\right)^{*}$. Since the grade of $\operatorname{Ext}^{1}(K, R)$ is at least $q$ we have that $\operatorname{Ext}^{j}\left(\operatorname{Ext}^{1}(K, R), R\right)$ is zero for $j=1$ through $q-1$. Thus $\operatorname{Ext}^{i}(V, R)$ is zero for $1 \leqslant i \leqslant q-2$. Using the usual CartanEilenberg construction for forming a projective resolution of the middle term of an exact sequence given resolutions of the ends, we obtain a diagram

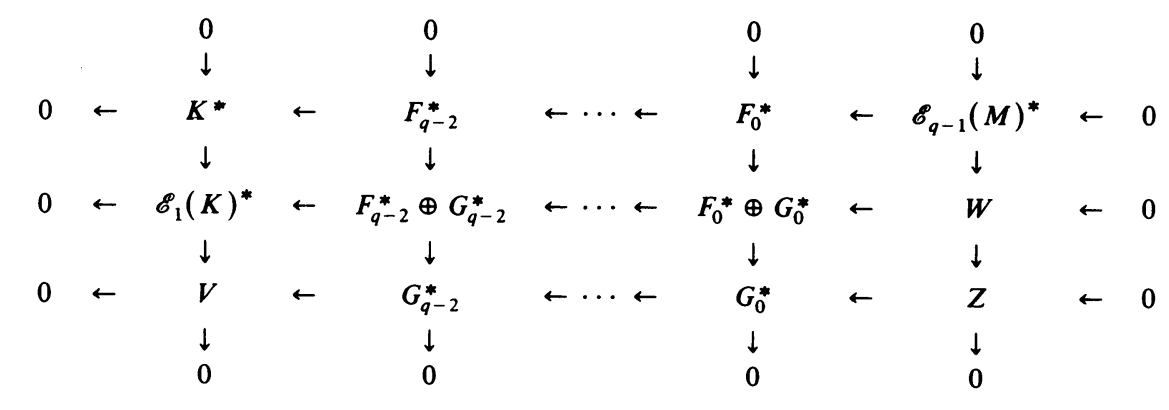


with exact rows and columns and with the $F_{i}{ }^{*}$ and $G_{i}^{*}$ free $R$-modules. We now dualize this diagram with respect to $R$ and use that

$$
0 \rightarrow K \rightarrow F_{q-2} \rightarrow \cdots \rightarrow F_{0} \rightarrow \mathscr{E}_{q-1}(M) \rightarrow 0
$$

is exact, that $V^{*} \cong R^{n}$ and that $\operatorname{Ext}^{i}(V, R)=0$ for $1 \leqslant i \leqslant q-2$. This yields the diagram

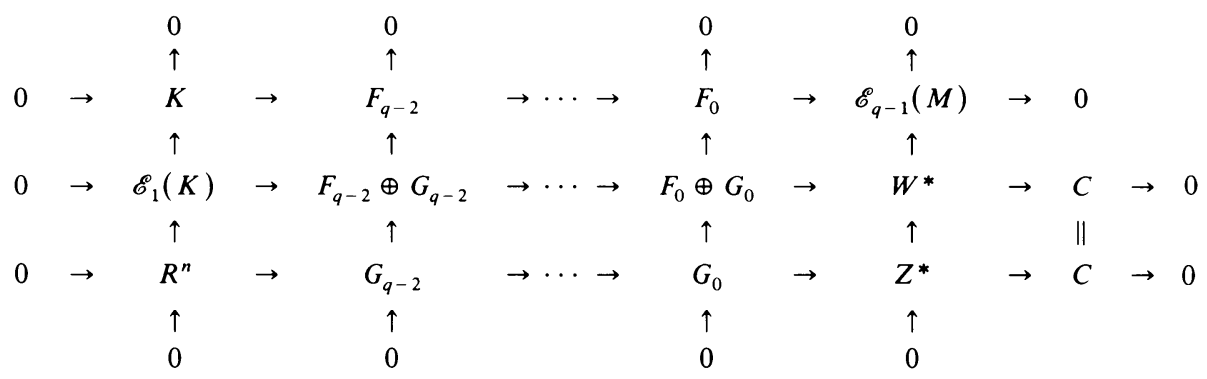

which has exact rows and columns. The cokernel $C$ is isomorphic to $\operatorname{Ext}^{q}\left(\operatorname{Ext}^{1}(K, R), R\right)$ which is in turn isomorphic to $\operatorname{Ext}^{q}\left(\operatorname{Ext}^{q}(M, R), R\right)$. We let $\mathscr{E}_{q}(M)$ be the image of $F_{0} \oplus G_{0}$ in $W^{*}$ and let $L$ be the image of $G_{0}$ in $Z^{*}$. Then the projective dimension of $L$ is at most $q-1$. We have an exact sequence

$$
0 \rightarrow L \rightarrow \mathscr{E}_{q}(M) \rightarrow \mathscr{E}_{q-1}(M) \rightarrow 0 .
$$

Then $\operatorname{Ext}^{q}\left(\mathscr{E}_{q}(M), R\right) \cong \operatorname{Ext}^{1}\left(\mathscr{E}_{1}(K), R\right)=0$ and $\operatorname{Ext}^{i}\left(\mathscr{E}_{q}(M), R\right)=0$ for $1 \leqslant i \leqslant$ $q$, by virtue of the dual exactness of the middle row of the above diagram. We let $\mathscr{K}_{q}(M)$ be the kernel of the map of $\mathscr{E}_{q}(M)$ onto $M$ via the composition of $\mathscr{E}_{q}(M) \rightarrow \mathscr{E}_{q-1}(M) \rightarrow M$. Therefore there is an exact sequence $0 \rightarrow L \rightarrow \mathscr{K}_{q}(M) \rightarrow$ $\mathscr{K}_{q-1}(M) \rightarrow 0$ and thus $\mathscr{K}_{q}(M)$ has projective dimension at most $q-1$ as desired.

REMARK 1.3. (a) The above short exact sequience $0 \rightarrow \mathscr{K}_{q}(M) \rightarrow \mathscr{E}_{q}(M) \rightarrow M \rightarrow 0$ will hereafter be referred to as a $q$ th presentation of $M$.

(b) If $M$ has finite projective dimension or if $M$ is locally free on the punctured spectrum of $R$ or if $R$ is Gorenstein, then the cohomology modules $\operatorname{Ext}^{i}(M, R)$ satisfy the grade assumptions of the theorem.

(c) If $M$ has projective dimension $q<\infty$, then the sequence $0 \rightarrow \mathscr{K}_{q}(M) \rightarrow$ $\mathscr{E}_{4}(M) \rightarrow M \rightarrow 0$ has $\mathscr{E}_{q}(M)$ free and thus is merely the beginning of a finite projective resolution of $M$. Thus the presentations $0 \rightarrow \mathscr{K}_{q}(M) \rightarrow \mathscr{E}_{q}(M) \rightarrow M \rightarrow 0$ begin with the Serre-Murthy exact sequence for $q=1$ and end with a free presentation of $M$ for $q$ equal to the projective dimension of $M$.

(d) The properties of a $q$ th presentation yield isomorphisms

$$
\operatorname{Ext}^{i}\left(\mathscr{K}_{q}(M), R\right) \cong \operatorname{Ext}^{i+1}(M, R) \text { for } 1 \leqslant i \leqslant q-1,
$$

and

$$
\operatorname{Ext}^{j}(M, R) \cong \operatorname{Ext}^{j}\left(\mathscr{E}_{q}(M), R\right) \text { for } j \geqslant q+1
$$


(e) If $R$ is Gorenstein and if $M$ is a reflexive $R$-module, then both $\mathscr{E}_{q}(M)$ and $\mathscr{K}_{q}(M)$ are reflexive. This follows because the cokernel $C$ is necessarily zero, since $\operatorname{Ext}^{q}(M, R)$ must have grade at least $q+1$ in case $M$ is reflexive.

The next results describe the uniqueness of the modules $\mathscr{E}_{q}(M)$ and $\mathscr{K}_{q}(M)$. The argument is in the same vein as many proofs of Schanuel's lemma.

THEOREM 1.4. If $0 \rightarrow \mathscr{K}_{q}(M) \rightarrow \mathscr{E}_{q}(M) \rightarrow M \rightarrow 0$ and $0 \rightarrow \mathscr{K}_{q}^{\prime}(M) \rightarrow \mathscr{E}_{q}^{\prime}(M) \rightarrow$ $M \rightarrow 0$ are both qth presentations of $M$, then $\mathscr{E}_{q}(M) \oplus \mathscr{K}_{q}^{\prime}(M)$ is isomorphic to $\mathscr{E}_{q}^{\prime}(M) \oplus \mathscr{K}_{q}(M)$.

Proof. We consider the following pullback diagram:

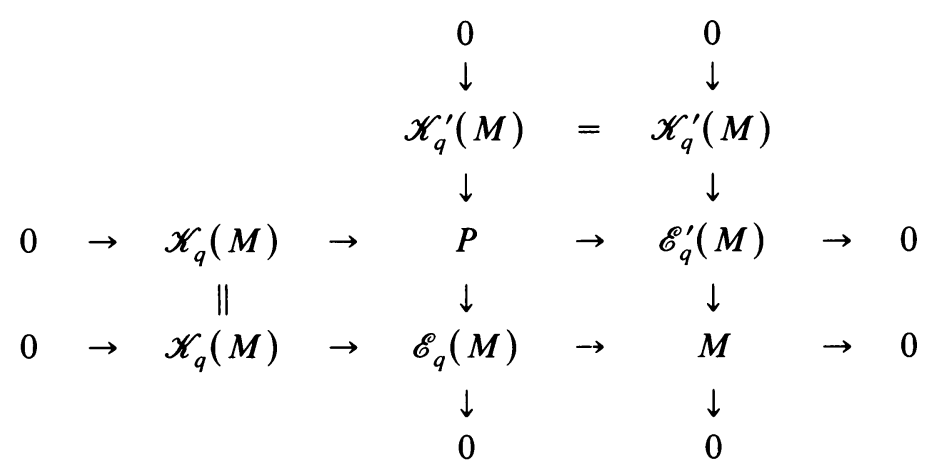

Clearly it suffices to show the middle row and column of the above diagram are split exact sequences. This is an immediate consequence of the following lemma.

LEMMA 1.5. Let $R$ be a local ring and suppose $A$ and $B$ are finitely generated $R$-modules such that the projective dimension of $A$ is less than $k$ and $\operatorname{Ext}^{i}(B, R)=0$ for $1 \leqslant i \leqslant k$. Then $\operatorname{Ext}^{i}(B, A)=0$ for $1 \leqslant i \leqslant k-\operatorname{pd} A$. (pd $A$ denotes the projective dimension of $A$.)

Proof. We proceed by induction on the projective dimension of $A$. The case pd $A=0$ is immediate.

Let pd $A=n>0$ and let $0 \rightarrow K \rightarrow F \rightarrow A \rightarrow 0$ be an exact sequence with $F$ a finitely generated free $R$-module. Applying the functor $\operatorname{Hom}(B,-)$ and taking the long exact sequence in cohomology yields the exact sequence

$$
\cdots \rightarrow \operatorname{Ext}^{i}(B, F) \rightarrow \operatorname{Ext}^{i}(B, A) \rightarrow \operatorname{Ext}^{i+1}(B, K) \rightarrow \cdots
$$

for each $i \geqslant 1$. If $1 \leqslant i \leqslant k-\operatorname{pd} A$, then $2 \leqslant i+1 \leqslant k-\operatorname{pd} K$. So by induction $\operatorname{Ext}^{i+1}(B, K)=0$. Now $\operatorname{Ext}^{i}(B, F)=0$ since $F$ is free and $\operatorname{Ext}^{i}(B, R)=0$. Thus $\operatorname{Ext}^{i}(B, A)=0$.

We now prove a stronger uniqueness which you would expect for the analogue of a free resolution.

Corollary 1.6. Let $0 \rightarrow \mathscr{K}_{q}(M) \rightarrow \mathscr{E}_{q}(M) \rightarrow M \rightarrow 0$ and $0 \rightarrow \mathscr{K}_{q}^{\prime}(M) \rightarrow \mathscr{E}_{q}^{\prime}(M)$ $\rightarrow M \rightarrow 0$ be two qth presentations for $M$. Then there are free $R$-modules $F, F^{\prime}, G$ and $G^{\prime}$ such that $\mathscr{K}_{q}(M) \oplus F \cong \mathscr{K}_{q}^{\prime}(M) \oplus F^{\prime}$ and $\mathscr{E}_{q}(M) \oplus G \cong \mathscr{E}_{q}^{\prime}(M) \oplus G^{\prime}$. 
Proof. We already know by Theorem 1.4 that $\mathscr{K}_{q}(M) \oplus \mathscr{E}_{q}^{\prime}(M) \cong \mathscr{K}_{q}^{\prime}(M) \oplus$ $\mathscr{E}_{q}(M)$. Further, it is enough to consider the case of $R$ complete, since if two finitely generated $R$-modules are isomorphic over the completion they are already isomorphic. Let $K_{1} \oplus \cdots \oplus K_{n}, E_{1} \oplus \cdots \oplus E_{m}, K_{1}^{\prime} \oplus \cdots \oplus K_{u}^{\prime}$ and $E_{1}^{\prime} \oplus \cdots \oplus E_{l}^{\prime}$ be the decompositions of $\mathscr{K}_{q}(M), \mathscr{E}_{q}(M), \mathscr{K}_{q}^{\prime}(M)$ and $\mathscr{E}_{q}^{\prime}(M)$ respectively. By the Krull-Schmidt Theorem for complete local rings and the above isomorphism, we have that $n+v=m+u$ and that the indecomposables from each side of the isomorphism are pairwise isomorphic. Furthermore, we can assume that $n \leqslant u$ and that the pairing is arranged so as to pair as many $K_{i}$ with $K_{j}^{\prime}$ as possible. If any $K_{i}$ is not paired with a $K_{j}^{\prime}$, then it is paired with an $E_{j}$. Hence it must have projective dimension less than $q$ while $\operatorname{Ext}^{s}\left(K_{i}, R\right)=0$ for $1 \leqslant s \leqslant q$. That is, in this case $K_{i}$ must be free and therefore just $R$ itself. Since the maximum number of $K_{i}$ 's were paired with the $K_{j}^{\prime}$ 's we must have that $\mathscr{K}_{q}(M) \oplus F \cong \mathscr{K}_{q}^{\prime}(M)$, where $F$ is free. Similarly $\mathscr{E}_{q}(M) \oplus F \cong \mathscr{E}_{q}^{\prime}(M)$, where $F$ is the same free module since the rank of the largest free summand on either side of the isomorphism $\mathscr{K}_{q}(M) \oplus \mathscr{E}_{q}^{\prime}(M) \cong$ $\mathscr{K}_{q}^{\prime}(M) \oplus \mathscr{E}_{q}(M)$ must be preserved.

If the $q$ th presentation for a module $M, 0 \rightarrow \mathscr{K}_{q}(M) \rightarrow \mathscr{E}_{q}(M) \rightarrow M \rightarrow 0$, has the property that there is a free $R$-module $F$ with $\mathscr{K}_{q}(M)=K_{q} \oplus F$ and $\mathscr{E}_{q}(M)=E_{q} \oplus F$ in which the map $\mathscr{K}_{q}(M) \rightarrow \mathscr{E}_{q}(M)$ induces an isomorphism of $F$ into $F$, then one may obtain a $q$ th presentation of the form $0 \rightarrow K_{q} \rightarrow E_{q} \rightarrow M \rightarrow 0$, that is, one may remove the free $R$-module $F$. In such a situation we refer to $\mathscr{K}_{q}(M)$ and $\mathscr{E}_{q}(M)$ as having a common free summand. Clearly at least one $q$ th presentation exists in which $\mathscr{K}_{q}(M)$ and $\mathscr{E}_{q}(M)$ have no common (nonzero) free summand. Such a $q$ th presentation is called a minimal $q$ th presentation. Our next theorem asserts that all $q$ th presentations are obtained from minimal ones by adding a common free summand to $\mathscr{K}_{q}(M)$ and $\mathscr{E}_{q}(M)$.

THEOREM 1.7. Let $0 \rightarrow \mathscr{K}_{q}(M) \rightarrow \mathscr{E}_{q}(M) \rightarrow M \rightarrow 0$ be a minimal qth presentation. If $0 \rightarrow K \rightarrow E \rightarrow M \rightarrow 0$ represents another qth presentation then $K$ and $E$ have $a$ common free summand $F$ such that $K \cong \mathscr{K}_{q}(M) \oplus F$ and $E \cong \mathscr{E}_{q}(M) \oplus F$.

Proof. It suffices to show, if $K$ and $E$ have no common free summand, then $K \cong \mathscr{K}_{q}(M)$ and $E \cong \mathscr{E}_{q}(M)$. By Corollary 1.6, we can write $\mathscr{K}_{q}(M)=K_{1} \oplus G_{1}$ and $K=K_{1} \oplus G_{2}$ so that $K_{1}$ has no nontrivial free summand and where $G_{1}$ and $G_{2}$ are free. Similarly, we have that $\mathscr{E}_{q}(M)=E_{1} \oplus F_{1}$ and $E=E_{1} \oplus F_{2}$, where $E_{1}$ has no nontrivial free summand and where $F_{1}$ and $F_{2}$ are free. Now consider the pullback diagram

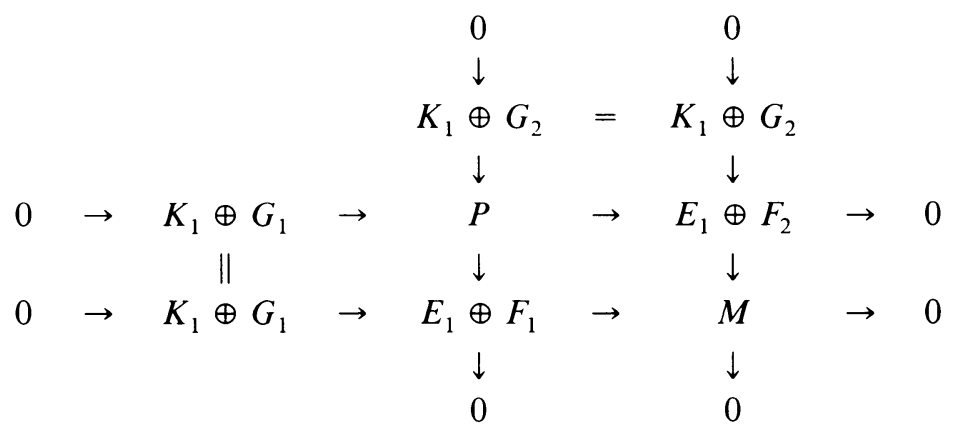


used in the proof of Theorem 1.4. The maps $K_{1} \oplus G_{1} \rightarrow F_{1}$ and $K_{1} \oplus G_{2} \rightarrow F_{2}$ necessarily have images in $\mathfrak{m} F_{1}$ and $\mathfrak{m} F_{2}$, respectively ( $\mathfrak{m}$ denotes the maximal ideal of $R$ ), since there are no common free summands. Thus the induced map $F_{1} \rightarrow F_{2}$, via the splitting of the middle column, must be surjective. By symmetry the induced map $F_{2} \rightarrow F_{1}$ must also be surjective. Thus $F_{1}$ and $F_{2}$ are isomorphic. From this fact together with Theorem 1.4 we conclude that $G_{1}$ and $G_{2}$ are isomorphic. Thus $\mathscr{E}_{q}(M) \cong E$ and $\mathscr{K}_{q}(M) \cong K$.

In the following theorem we collect some properties of these presentations.

THEOREM 1.8. Let $R$ be a local Cohen-Macaulay ring and let $M$ be a finitely generated $R$-module.

(a) If $M$ has finite projective dimension less than or equal to $q$ and if $0 \rightarrow \mathscr{K}_{q}(M) \rightarrow$ $\mathscr{E}_{q}(M) \rightarrow M \rightarrow 0$ is a qth presentation of $M$, then $\mathscr{E}_{q}(M)$ is free and $\mathscr{K}_{q}(M)$ is the module of relations on $M$.

(b) IF $\operatorname{Ext}^{q+1}(M, R)=0$, then a qth presentation of $M$ is also $a(q+1)$ st presentation.

(c) If $f: M \rightarrow N$ is a homomorphism of $R$-modules, then for each $q$, there is an induced commutative diagram:

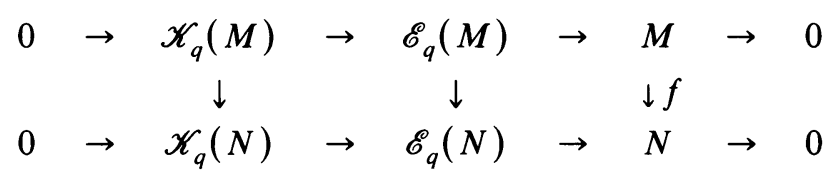

(d) If $M$ represents a vector bundle on the punctured spectrum of $R$, then each qth presentation $0 \rightarrow \mathscr{K}_{q}(M) \rightarrow \mathscr{E}_{q}(M) \rightarrow M \rightarrow 0$ is an exact sequence of vector bundles.

(e) If for some $q \geqslant 2$ we have that $\mathscr{K}_{q}(M)$ is free, then $\operatorname{Ext}^{i}(M, R)=0$ for $2 \leqslant i \leqslant q$.

Proof. Straightforward (see Remarks 1.3 and Lemma 1.5).

Let $M$ be a module of finite projective dimension $n$ over $R$. As noted above the presentation $0 \rightarrow \mathscr{K}_{n}(M) \rightarrow \mathscr{E}_{n}(M) \rightarrow M \rightarrow 0$ is a free presentation of $M$. Moreover, for $0 \leqslant i \leqslant j \leqslant n$ and taking $\mathscr{E}_{0}(M)=M, \mathscr{K}_{0}(M)=0$, one may obtain commutative triangles (see beginning of the proof of Theorem 1.2)

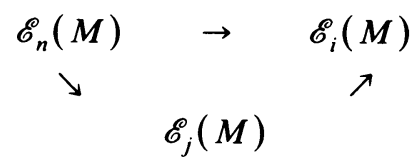

where the maps are surjective (however, the presentations are not in general minimal in this setup). One also obtains corresponding commutative triangles

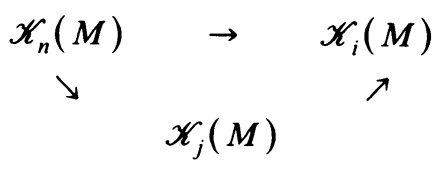


with surjective maps. In this fashion we obtain a filtration based on cohomology of a (nonminimal, in general) free presentation of $M$. We end this section with two corollaries resulting from this filtration.

COROllaRY 1.9. Let $R$ be a Gorenstein ring of dimension $n$ and let $M$ be a finitely generated $R$-module of depth $d$. Then $\operatorname{Ext}^{i+1}(M, R) \cong \operatorname{Ext}^{i}\left(\mathscr{K}_{n-d}(M), R\right)$ for $1 \leqslant i$ $\leqslant n-d-1$. That is the cohomology modules $\operatorname{Ext}^{i}(M, R)$, for $2 \leqslant i \leqslant n-d$, are isomorphic to those of a module of finite projective dimension.

Proof. For $R$ Gorenstein of dimension $n$ and $M$ of depth $d$, the only possible nonzero $\operatorname{Ext}^{i}(M, R)$ lie in the range $1 \leqslant i \leqslant n-d$. Then $\operatorname{Ext}^{i}\left(\mathscr{E}_{n-d}(M), R\right)=0$ for $i>0$ while $\operatorname{Ext}^{i}\left(\mathscr{K}_{n-d}(M), R\right)$ is isomorphic to $\operatorname{Ext}^{i+1}(M, R)$ for $i>0$ (see 1.3(d)).

Our remaining corollary contains a weaker version of our result [6, Theorem 2.1]. This is moderately surprising since our proof there uses our syzygy theorem [4] which in turn uses Hochster's big Cohen-Macaulay modules [8], while the result below only uses Theorem 1.2. However, it should be stressed that we do not recover the full generality of [6, Theorem 2.1] but only the case of a vector bundle and without the minimality implicit in the earlier result.

In Corollary 1.10 as well as the next section we need to make use of Matlis duality. For an $R$-module $L$, the Matlis dual of $L$ is $\operatorname{Hom}(L, I)$, where $I$ is the injective envelope of the residue field of $R$. We use the notation $L^{v}$ to indicate the dual module $\operatorname{Hom}(L, I)$. If $L$ is an Artinian module, then $L$ is naturally isomorphic to $L^{\text {"n'}}$.

COROLlaRY 1.10. Let $R$ be a regular local ring of dimension $n$ with $n \geqslant 4$. Let $M$ be a kth syzygy which is not $a(k+1)$ th syzygy, for $2 \leqslant k \leqslant n-2$, which represents $a$ vector bundle on the punctured spectrum of $R$. Then in the $(n-k-1)$ th presentation $\mathscr{K}_{n-k-1}(M)$ is $a(k+2)$ th syzygy and $\mathscr{E}_{n-k-1}(M)$ is a kth syzygy of $\operatorname{Ext}^{k-1}\left(M^{*}, R\right)$.

REMARK. Unlike [6, Theorem 2.1] $\mathscr{E}_{n-k-1}(M)$ may not be the minimal $k$ th syzygy of $\operatorname{Ext}^{k-1}\left(M^{*}, R\right)$.

Proof. Since $M$ represents a vector bundle on the punctured spectrum of $R$, each $\operatorname{Ext}^{i}(M, R)$ has finite length. The projective dimension of $M$ is $n-k$. Thus by Theorem 1.2 and Theorem 1.8, $\mathscr{K}_{n-k-1}(M)$ and $\mathscr{E}_{n-k-1}(M)$ also represent vector bundles. Furthermore $\mathscr{K}_{n-k-1}(M)$ has projective dimension $n-k-2$ and $\mathscr{E}_{n-k-1}(M)$ has a unique nonvanishing $\operatorname{Ext}^{i}$, namely $\operatorname{Ext}^{n-k}\left(\mathscr{E}_{n-k-1}(M), R\right)$. Hence $\mathscr{K}_{n-k-1}(M)$ is a $n-(n-k-2)=(k+2)$ th syzygy and $\mathscr{E}_{n-k-1}(M)$ is a $k$ th syzygy of $\operatorname{Ext}^{n-k}\left(\mathscr{E}_{n-k-1}(M), R\right)^{\text {" }}$ (see discussion of Horrocks and Matlis duality in §2) which is isomorphic to $\operatorname{Ext}^{n-k}(M, R)^{v}$. However, the duality of Horrocks [9] yields that $\operatorname{Ext}^{n-k}(M, R)^{v}=\operatorname{Ext}^{k-1}\left(M^{*}, R\right)$, which is nonzero because $M$ is not a $(k+1)$ th syzygy.

2. Lifting vector bundles. Throughout this section $R$ will denote a regular local ring of dimension $n$ with $n \geqslant 3$. We also assume that $R$ contains a field in order that we may apply our results in $[4,5]$. The $R$-module $M$ will be finitely generated and 
reflexive and will denote a vector bundle on the punctured spectrum of $R$, that is, $M_{P}$ is a free $R_{P}$-module for each nonmaximal prime ideal $P$ of $R$. We shall use $S$ to denote a regular local ring with parameter $t$ such that $S / t S=R$.

If there is a finitely generated reflexive $S$-module $M$, which is a vector bundle on the punctured spectrum of $S$, such that $\left(M^{\prime} / t M^{\prime}\right)^{* *}$ is isomorphic to $M$ (the double dual here is taken with respect to $R$ ), then we say that $M$ lifts as a bundle to $S$. Horrocks [10] has asked if a bundle which lifts to arbitrarily high dimension must be trivial, that is, must the module $M$ be free? (For an affirmative answer in the case of projective space see $[2,12,14]$.) In [5] we have established this fact in case $\operatorname{Ext}^{i}(M, R)$ vanishes for $i=1$ or $i=2$. In this section we extend this result to include any $i$ for $2 \leqslant i \leqslant n-3$. Hence in view of the duality of Horrocks [9] and our result [5, Theorem 2.5] we establish an affirmative answer to Horrocks' question in case any cohomology $\operatorname{Ext}^{i}(M, R)$ vanishes for some $i$ in the range $1 \leqslant i \leqslant n-2$. Often we shall express these statements in terms of the sheaf cohomology of $M$ on the punctured spectrum (viewing $M$ as a vector bundle), and so we remind the reader of Horrocks' article [9] and specifically of the isomorphisms

$$
H^{i}(M) \cong \operatorname{Ext}^{i}\left(M^{*}, R\right) \text { and } H^{n-j-1}\left(M^{*}\right) \cong H^{j}(M)^{v},
$$

where $(-)^{v}$ denotes the Matlis dual (see the end of $\$ 1$ for a definition of Matlis duality).

Our technique is to show that a lifting of $M$ together with the vanishing of $\operatorname{Ext}^{i}(M, R)$ for some $i$ with $2 \leqslant i \leqslant n-3$, induces a lifting of $\mathscr{K}_{i}(M)$. However, in this situation $\mathscr{K}_{i}(M)$ is at least a third syzygy and thus our result [5, Theorem 2.5] may be applied to $\mathscr{K}_{i}(M)$. Furthermore we provide bounds derived from intrinsic data on $M$ as to how far one may expect to lift $M$ as a bundle.

The notation $\bar{K}$ indicates the reduction of an $S$-module $K$ modulo the parameter $t$, i.e., $\bar{K}=K / t K$.

Lemma 2.1. Suppose that the reflexive $S$-module $L$ lifts $M$ as a bundle to $S$. Let $0 \rightarrow \mathscr{K}_{q+1}(L) \rightarrow \mathscr{E}_{q+1}(L) \rightarrow L \rightarrow 0$ be $a(q+1)$ th presentation of $L$ over $S$, where $1 \leqslant q \leqslant n-2$.

(a) If $\overline{\mathscr{K}_{q+1}(L)}$ and $\overline{\mathscr{E}}_{q+1}(L){ }^{* *}$ (the double dual with respect to $R$ ) have a common free $R$-summand, then this free summand lifts to a common free $S$-summand of $\mathscr{K}_{q+1}(L)$ and $\mathscr{E}_{q+1}(L)$.

(b) If $\operatorname{Ext}_{R}^{q}(M, R)=H^{q}\left(M^{*}\right)=0$ for some $q$ with $1 \leqslant q \leqslant n-3$, then $\operatorname{Ext}_{S}^{i}(L, S)=H^{i}\left(L^{*}\right)=0$ for $i=q, q+1$ and the sequence

$$
0 \rightarrow \overline{\mathscr{K}_{q+1}(L)} \rightarrow{\overline{\mathscr{E}_{q+1}}(L)}^{* *} \rightarrow M \rightarrow 0
$$

is a qth presentation of $M$ over $R$.

Proof. Since $\mathscr{K}_{q+1}(L)$ necessarily has depth at least 4 (see Theorem 1.2) it follows that $\overline{\mathscr{K}_{q+1}(L)}$ is necessarily $R$-reflexive and that the sequence

$$
0 \rightarrow \overline{\mathscr{K}_{q+1}(L)} \rightarrow{\overline{\mathscr{E}_{q+1}(L)}}^{* *} \rightarrow M \rightarrow 0
$$


is exact (note $\bar{L}^{* *} \cong M$ ). In regard to part (b), the vanishing of the cohomology $H^{i}\left(L^{*}\right)$ for $i=q, q+1$ is just a result of the long exact sequence for cohomology (see $[5,1.6])$. For the same reason it follows that $\left.\operatorname{Ext}_{R}^{i} \overline{\mathscr{E}}_{q+1}(L){ }^{* *}, R\right)=0$ for $1 \leqslant i \leqslant q$. Since $\operatorname{Ext}_{S}^{i}(L, S)=0$ for $i=q, q+1$ it also follows that $\mathscr{K}_{q+1}(L)$ has projective dimension at most $q-1$ over $S$. However, the projective dimension of $\mathscr{K}_{q+1}(L)$ over $S$ is the same as the projective dimension of $\overline{\mathscr{K}_{q+1}(L)}$ over $R$. Part (b) now follows from the uniqueness result (Theorem 1.4). Returning to part (a), we observe that a common free summand $F$ of $\overline{\mathscr{K}_{q+1}(L)}$ and $\overline{\mathscr{E}_{q+1}(L)}{ }^{*}$ as described by the commutative triangle

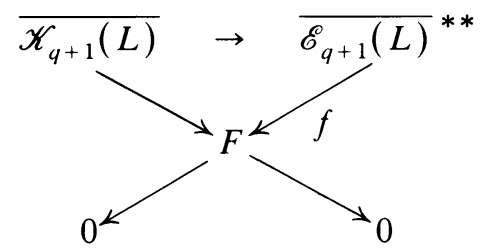

actually yields an identical one in which the $R$-double dual of $\overline{\mathscr{E}_{q+1}(L)}$ is removed. For the extension modules $\mathscr{E}_{q+1}(L)$ one always has an epimorphism $\operatorname{Hom}\left(\mathscr{E}_{q+1}(L), S\right) \rightarrow \operatorname{Hom}_{R}\left(\mathscr{E}_{q+1}(L), R\right)$, since one always has that the cohomology module $\operatorname{Ext}_{S}^{1}\left(\mathscr{E}_{q+1}(L), S\right)=0$. Therefore the map $f: \overline{\mathscr{E}_{q+1}(L)} \rightarrow F$ lifts to an epimorphism $g: \mathscr{E}_{q+1}(L) \rightarrow G$, where $G / t G=F$. Further we note that $g$ maps $\mathscr{K}_{q+1}(L)$ onto $G$, by Nakayama's Lemma, since $f$ takes $\mathscr{K}_{q+1}(L)$ onto $F$. Thus part (a) is also established.

Corollary 2.2 (NOTAtion AS ABOVE). If $0 \rightarrow \mathscr{K}_{q}(M) \rightarrow \mathscr{E}_{q}(M) \rightarrow M \rightarrow 0$ and $0 \rightarrow \mathscr{K}_{q+1}(L) \rightarrow \mathscr{E}_{q+1}(L) \rightarrow L \rightarrow 0$ are minimal presentations over $R$ and $S$, respectively, and if $H^{q}\left(M^{*}\right)=0$, then $H^{q}\left(L^{*}\right)=0$ and $\mathscr{K}_{q+1}(L)$ lifts $\mathscr{K}_{q}(M)$ as a bundle.

Proof. The proof is an immediate consequence of the uniqueness result Theorem 1.7 and Lemma 2.1. For in this situation, Lemma 2.1 guarantees (when $H^{q}\left(L^{*}\right)=0$ ) that $0 \rightarrow \mathscr{K}_{q+1}(L) \rightarrow \mathscr{E}_{q+1}(L) \rightarrow L \rightarrow 0$ is a minimal $(q+1)$ th presentation over $S$ if and only if $0 \rightarrow \overline{\mathscr{K}_{q+1}(L)} \rightarrow \overline{\mathscr{E}}_{q+1}(L){ }^{* *} \rightarrow M \rightarrow 0$ is a minimal $q$ th presentation over $R$.

In order to apply our result [5, Theorem 2.5$]$ we would like to obtain an estimate of the ranks of the $\mathscr{K}_{q}(M)$ in terms of more familiar invariants.

Lemma 2.3. Let $\rho_{i}(M)$ be the rank of the ith syzygy of $\operatorname{Ext}^{i}(M, R)$ for $i \geqslant 0$ and let $0 \rightarrow \mathscr{K}_{q}(M) \rightarrow \mathscr{E}_{q}(M) \rightarrow M \rightarrow 0$ be a minimal qth presentation for $M$. Then

$$
\operatorname{rank}\left(\mathscr{K}_{q}(M)\right) \leqslant \sum_{i=1}^{q} \rho_{i}(M) \text { for } q \geqslant 1 .
$$

Proof. We induct on $q$. If $q=1$, then $\mathscr{K}_{1}(M)$ is isomorphic to $R^{\mu}$, where $\mu$ is the number of generators of $\operatorname{Ext}^{1}(M, R)$. However, since $\operatorname{Ext}^{1}(M, R)$ is torsion, then $\mu$ is also the rank of the first syzygy of $\operatorname{Ext}^{1}(M, R)$. 
If $q>1$, we appeal to the inductive description of the filtration (see discussion after Theorem 1.8 as well as the proof of Theorem 1.2) to obtain a commutative diagram

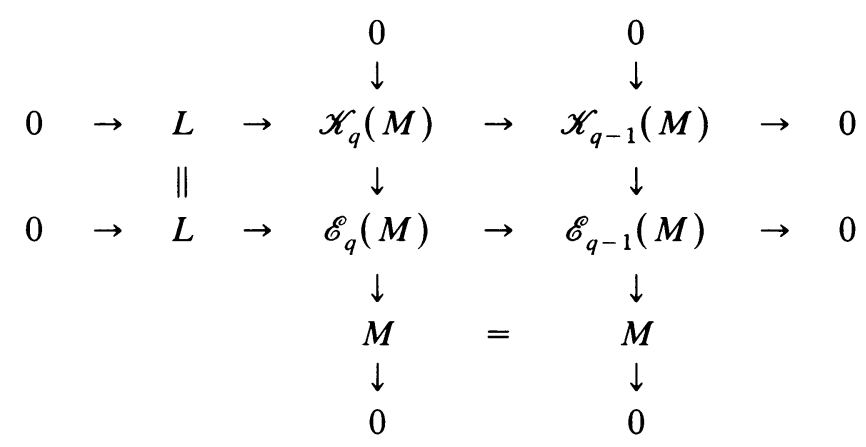

in which $L$ has at most one nonzero cohomology, namely $\operatorname{Ext}^{q-1}(L, R)$. This property of $L$ is inherent in the inductive step in the proof of Theorem 1.2 (particularly in view of the dual exactness properties of the complex $G$ ). While we may assume that the $(q-1)$ th presentation in the above diagram is minimal we cannot assume the same about the $q$ th presentation (middle column), since it may be necessary to add on a free module in order to keep the rows exact. However, the desired inequality will follow from the subsequent argument since the rank of the "minimal $\mathscr{K}_{q}(M)$ " cannot be larger that that of $\mathscr{K}_{q}(M)$ in the diagram.

Returning to the module $L$ we have that

$$
\operatorname{Ext}^{q-1}(L, R) \cong \operatorname{Ext}^{q-1}\left(\mathscr{K}_{q}(M), R\right) \cong \operatorname{Ext}^{q}(M, R) \text { for } q \geqslant 2 .
$$

Since $\operatorname{Ext}^{1}\left(\mathscr{E}_{q-1}(M), R\right)=0$, the sequence $0 \rightarrow \mathscr{E}_{q-1}(M)^{*} \rightarrow \mathscr{E}_{q}(M)^{*} \rightarrow L^{*} \rightarrow 0$ is exact. As a consequence we may assume that $L^{*}$ and therefore also $L$ is constructed without nontrivial free summands. Thus, if $L$ is nonzero, then $L$ is a bundle with one nonzero cohomology module, $\operatorname{Ext}^{q-1}(L, R)$. Therefore $L^{*}$ is a $q$ th syzygy for $\operatorname{Ext}^{q-1}(L, R)=\operatorname{Ext}^{q}(M, R)$. But $L$ and $L^{*}$ have the same rank. It follows from the exact sequence $0 \rightarrow L \rightarrow \mathscr{K}_{q}(M) \rightarrow \mathscr{K}_{q-1}(M) \rightarrow 0$ that rank $\mathscr{K}_{q}(M)=\operatorname{rank} L+$ rank $\mathscr{K}_{q-1}(M)$. Finally by induction we obtain the desired inequality rank $\mathscr{K}_{q}(M)$ $\leqslant \sum_{i=1}^{q} \rho_{i}(M)$, where $\mathscr{K}_{q}(M)$ is minimal.

We can now establish the bounds on the lifting of a vector bundle $M$ having a vanishing cohomology module. We keep the notation of the previous discussion.

THEOREM 2.4. Let $M$ be a nonfree, reflexive $R$-module which represents a vector bundle on the punctured spectrum of $R$. If $H^{q}\left(M^{*}\right) \cong \operatorname{Ext}^{q}(M, R)=0$ for some $q$ with $1 \leqslant q \leqslant \operatorname{dim} R-2$, then $M$ can be lifted at most $\sum_{i=0}^{q-1} \rho_{i}(M)$ times.

Proof. If $q=0$ or 1 , then the result follows from our calculations in [5, Theorems 2.5 and 2.6]. So suppose that neither $\operatorname{Ext}^{1}(M, R)$ nor $\operatorname{Ext}^{2}(M, R)$ are zero, but that $\operatorname{Ext}^{q}(M, R)$ is zero for some $q>2$. By duality with $M^{*}$ and the case $q=0$ we may assume that $q \leqslant \operatorname{dim} R-4$. By Theorem 1.8(c), $\mathscr{K}_{q}(M)$ cannot be free. However, by Corollary 2.2, we have that $\mathscr{K}_{q}(M)$ lifts every time that $M$ does. From Lemma 2.3, $\mathscr{K}_{q}(M)$ has rank less than or equal to $\sum_{i=0}^{q-1} \rho_{i}(M)$ (note $\rho_{q}(M)=0$ ) and has depth at 
least $\operatorname{dim} R-q+1 \geqslant 3$. Again we appeal to [5, Theorem 2.5] and find that $\mathscr{K}_{q}(M)$ cannot be lifted more than $\left[\sum_{i=0}^{q-1} \rho_{i}(M)\right]-(\operatorname{dim} R-q+1)$ times. Thus our argument is complete.

We remark that one could obtain finer bounds depending on which (or how many) $\operatorname{Ext}^{q}(M, R)$ vanish. However these can readily be obtained if needed by combining Theorems 2.5 and 2.6 of [5] together with Corollary 2.2 and Lemma 2.3 in individual cases. We also remark that $M$ lifts if and only if $M^{*}$ does, and moreover the cohomology modules are dual via Matlis duality. Thus one may restrict to the case of $\operatorname{Ext}^{q}(M, R)=0$ for $q$ at most $\frac{1}{2}(\operatorname{dim} R+1)$. This suggests that perhaps the number $\sum_{i=0}^{h} \rho_{i}(M)$ is an upper bound for the number of times $M$ can be lifted for general $M$, where $h$ is the greatest integer in $\frac{1}{2}(\operatorname{dim} R+1)$.

ADDED IN PROOF. We have recently learned that the existence of $q$ th presentations (Theorem 1.2) and also Corollary 1.7 were first noted by Auslander and Bridger [1].

\section{REFERENCES}

1. M. Auslander and M. Bridger, Stable module theory, Mem. Amer. Math. Soc. No. 94 (1969).

2. W. Barth and A. Van de Ven, A decomposability criterion for algebraic 2-bundles on projective spaces, Invent. Math. 25 (1974), 91-106.

3. W. Bruns, E. G. Evans and P. Griffith, Syzygies, ideals of height two and vector bundles, J. Algebra 67 (1980), 143-162.

4. E. G. Evans and P. Griffith, The syzygv problem, Ann. of Math. 114 (1981), 323-333.

5. L_ Lifting syzygies and extending algebraic vector bundles, Amer. J. Math. (to appear).

6. Syzygies of critical rank, Quart. J. Math. 35 (1984), 393-402.

7. R. Hartshorne, Algebraic vector bundles on projective spaces: A problem list, Topology 18 (1979), $117-128$.

8. M. Hochster, Topics in the homological theory of modules over commutative rings, C.B.M.S. Regional Conf. Ser. Math., no. 24, Amer. Math. Soc., Providence, R. I., 1976.

9. G. Horrocks, Vector bundles on the punctured spectrum of a local ring, Proc. London Math. Soc. (3) 14 (1964), 689-713.

10. On extending vector bundles over projective space, Quart. J. Math. 17 (1966), 14-18.

11. P. Murthy, Generators for certain ideals in regular rings of dimension three, Comment. Math. Helv. 47 (1972), 179-184.

12. E. Sato, On the decomposability of infinitely extendable vector bundles on projective spaces and Grassmann varieties, J. Math. Kyoto Univ. 17 (1977), 127-150.

13. J.-P. Serre, Sur les modules projectifs, Sém. Dubriel-Pisot 2 (1960/1961), 13.

14. A. N. Tyurin, Finite dimensional vector bundles over infinite varieties, Izv. Akad. Nauk Ser. Mat. 40 (1976), 1248-1268; English transl., Math. U.S.S.R. Izv. 10 (1976), 1187-1204.

Department of Mathematics, University of Illinois, Urbana, Illinois 61801 